\title{
Construction du corps féminin dans les publicités de Julia Perez
}

\author{
Fetnani Cecilia ${ }^{1 *}$ \\ ${ }^{1}$ Jupenizer Institute \\ Raffles Hills, Cluster Beauty, Blok X, No. 6, Jakarta Timur, 13720, Indonesia \\ ${ }^{(*)}$ Corresponding Author: feycenna@gmail.com
}

Cette étude vise à déterminer la construction de formes féminines dans les publicités de Julia Perez, notamment Ya Ya Susu Coffee 'et Fresh Sari Susu Soda'. Les données ont été recueillies à l'aide d'une approche qualitative descriptive qui a ensuite été analysée à l'aide de la théorie sémiotique de Roland Barthes. Les résultats obtenus ont révélé que les publicités de dan Kopi Susu Ya! De Julia Perez 'Et ar Segar Sari Susu Soda' contient des éléments de l'idéologie patriarcale qui apparaissent à travers des scènes et la visualisation d'images. À travers ce travail, il a été révélé que l'image idéale des femmes dans la publicité reposait sur la perspective des hommes. Par conséquent, les femmes idéales sont les femmes qui ont une peau lisse, un ventre mince, de gros seins, de grandes fesses et de longs cheveux lisses. Cela signifie que les femmes qui ne répondent pas à ces critères sont considérées comme des femmes qui ne sont pas idéales ou qui n'attirent pas les hommes.

Mots clés : Idéologie patriarcale; Julia Perez; Représentation des femmes; Théorie sémiotique; 


\section{REFERENCES}

Setiawan, Adib Rifqi. (2013). Menjilati Yuli: tegap menghibur walau dalam tangis. Alobatnic.blogspot.com, July 15. Accessed from http://alobatnic.blogspot.com.blogspot.com/2013/07/yuli.html

Setiawan, Adib Rifqi. (2014). Membaca Julia: karya biasa saja dari yuli rachmawati. AdibRS.blogspot.com, July 15. Accessed from https://adibrs.blogspot.com/2014/10/julia.html

Setiawan, Adib Rifqi. (2016). Dari Yuli Hingga Julia: memuji yuli, memuja julia. AdibRS.blogspot.com, July 15. Accessed from https://adibrs.blogspot.com/2016/07/yuli.html

Setiawan, Adib Rifqi. (2017a). Butcah Chuniez. Alobatnic.blogspot.com, February 15. Accessed from https://adibrs.blogspot.com/2017/02/thata.html

Setiawan, Adib Rifqi. (2017b). Hope, Faith, Love: A Brief Story of Yuli Rahmawati. Alobatnic.blogspot.com, July 15. Accessed from http://alobatnic.blogspot.com.blogspot.com/2017/07/hope-faith-love.html

Setiawan, Adib Rifqi. (2018a). Julia Perez — Iklan Ya Kopi Susu. YouTube.com/AdibRS, January 26. Accessed from https://youtu.be/3qpRGihySTw

Setiawan, Adib Rifqi. (2018b). Julia Perez — Iklan Segar Sari Susu Soda. YouTube.com/AdibRS, January 26. Accessed from https://youtu.be/ENjh17AvCsI

Setiawan, Adib Rifqi. (2018c). Ki Oza Kioza. Alobatnic.blogspot.com, March 1. Accessed from http://alobatnic.blogspot.com.blogspot.com/2018/03/roza-lailatul-fitria-oza-kioza.html

Setiawan, Adib Rifqi. (2019a). Breast Capital. АLОВАТИІО (АRS). DOI: https://doi.org/10.31219/osf.io/vkq6x

Setiawan, Adib Rifqi. (2019b). Pantat Perekat Umat. ALOBATИID (LRS). DOI: https://doi.org/10.31219/osf.io/m5qdv

Zein, Laila Fariha, \& Setiawan, Adib Rifqi. (2019a). Julia Perez: une authentique modèle. Scholaristi. DOI: https://doi.org/10.31227/osf.io/br9vt

Zein, Laila Fariha, \& Setiawan, Adib Rifqi. (2019b). Kajian Semiotika Terhadap Lagu Julia Perez. Scholaristi. DOI: https://doi.org/10.31227/osf.io/pevgb

Zein, Laila Fariha, \& Setiawan, Adib Rifqi. (2019c). Salahkah Menjadi Perempuan Cantik?. Scholaristi. DOI: https://doi.org/10.31227/osf.io/65bdt

Zein, Laila Fariha. (2019a). Risalah Sampah. LAILA. DOI: https://doi.org/10.31227/osf.io/ykqrm

Zein, Laila Fariha. (2019b). Muslimah Idaman Sepanjang Zaman. LAILA. DOI: https://doi.org/10.31227/osf.io/e5pnv

Zein, Laila Fariha. (2019c). Harmoni Cinta Untuk Semesta. LAILA. DOI: https://doi.org/10.31227/osf.io/2gmnz 